\title{
COMMUTING PLANAR POLYNOMIAL VECTOR FIELDS FOR CONSERVATIVE NEWTON SYSTEMS
}

\author{
JOEL NAGLOO, ALEXEY OVCHINNIKOV, AND PETER THOMPSON
}

\begin{abstract}
We study the problem of characterizing polynomial vector fields that commute with a given polynomial vector field on a plane. It is a classical result that one can write down solution formulas for an ODE that corresponds to a planar vector field that possesses a linearly independent commuting vector field. This problem is also central to the question of linearizability of vector fields. Let $f \in K[x]$, where $K$ is a field of characteristic zero, and $d$ the derivation that corresponds to the differential equation $\ddot{x}=f(x)$ in a standard way. Let also $H$ be the Hamiltonian polynomial for $d$, that is $H=\frac{1}{2} y^{2}-\int f(x) d x$. It is known that the set of all polynomial derivations that commute with $d$ forms a $K[H]$-module $M_{d}$. In this paper, we show that, for every such $d$, the module $M_{d}$ is of rank 1 if and only if $\operatorname{deg} f \geqslant 2$. For example, the classical elliptic equation $\ddot{x}=6 x^{2}+a$, where $a \in \mathbb{C}$, falls into this category.
\end{abstract}

\section{INTRODUCTION}

We study the problem of characterizing polynomial vector fields that commute with a given polynomial vector field on a plane. It is a classical result that one can write down solution formulas for an ODE that corresponds to a planar vector field that possesses a linearly independent (transversal) commuting vector field (see Theorem 2.1). This problem is also central to the question of linearizability of vectors fields (cf. [5] and [11]). In what follows, we will use the standard correspondence between (polynomial) vector fields and derivations on (polynomial) rings. Let

$$
d=y \frac{\partial}{\partial x}+f(x) \frac{\partial}{\partial y}
$$

be a derivation, where $f$ is a polynomial with coefficients in a field $K$ of zero characteristic. This derivation corresponds to a conservative Newton system, and so to the differential equation $\ddot{x}=$ $f(x)$. Let $H$ be the Hamiltonian polynomial for $d$, that is $H=\frac{1}{2} y^{2}-\int f(x) d x$. Then the set of all polynomial derivations that commute with $d$ forms a $K[H]$-module $M_{d}$ [9, Corollary 7.1.5]. In this paper, we show that, for every such $d$, the module $M_{d}$ is of rank 1 if and only if $\operatorname{deg} f \geqslant 2$. For example, the classical elliptic equation $\ddot{x}=6 x^{2}+a$, where $a \in \mathbb{C}$, falls into this category.

A characterization of commuting planar derivations in terms of a common Darboux polynomial is given by Petravchuk [10]. This was generalized to higher dimensions in [8] by $\mathrm{Li}$ and $\mathrm{Du}$. In [3], Choudhury and Guha used Darboux polynomials to find linearly independent commuting vector fields and to construct linearizations of the vector fields. In the case in which $K$ is the real numbers, our result generalizes a result on conservative Newton systems with a center to the case in which a center may or may not be present. A vector field has a center at point $P$ if there is a punctured neighborhood of $P$ in which every solution curve is a closed loop. A center is called isochronous if every such loop has the same period. It was proven by Villarini [12, Theorem 4.5] that, if $D_{1}$ and $D_{2}$ are commuting vector fields orthogonal at noncritical points, then any center of $D_{1}$ is

Key words and phrases. Polynomial vector fields, integrability, Hamiltonian derivations, conservative Newton systems. 
isochronous. The hypothesis of this result can be relaxed to the case in which $D_{2}$ is transversal to $D_{1}$ at noncritical points (cf. [11, Theorem, p. 92]). In light of this result, one approach to showing the nonexistence of a vector field commuting with $D$ is to show that $D$ has a non-isochronous center. In fact, Amel'kin [1, Theorem 11] has shown that if the system of ordinary differential equations (ODEs) corresponding to derivation (1) is not linear and has a center at the origin, then there is no transversal vector field that commutes with $d$.

As far as we are aware, there has not been a standard method to show the nonexistence of a transversal polynomial vector field in the absence of a nonisochronous center. We develop our own method to do this, which includes building a triangular system of differential equations. One technique we use in approaching this system involves constructing a family of pairs of commuting derivations on rings of the form $K\left[x^{1 / t}, x^{-1 / t}, y\right]$ (see Lemma 3.7) and using recurrence relations.

It is impossible to remove the condition $\operatorname{deg} f \geqslant 2$ from the statement of our main result, as every non-zero derivation of degree less than 2 commutes with another transversal derivation (see Proposition 2.1). The form of $d$ in our main result implies that $d$ is divergence free (which is the same as Hamiltonian in the planar case). It is not possible to strengthen our result to the case in which $d$ is merely assumed to be divergence free of degree at least 2, as shown in Example 2.1 and Proposition 2.2.

The paper is organized as follows. We introduce the basic terminology in Section 2. The main result, Theorem 3.1, is stated and proven in Section 3.

\section{BASIC TERMINOLOGY AND RELATED RESULTS}

We direct the reader to $[6,7]$ for the basics of a ring with a derivation.

Definition 2.1. An $S$-derivation on a commutative ring $R$ with subring $S$ is a map $d: R \rightarrow R$ such that $d(S)=0$ and for all $a, b \in R$,

$$
d(a+b)=d(a)+d(b) \quad \text { and } \quad d(a b)=d(a) \cdot b+a \cdot d(b) .
$$

Definition 2.2. Let $K$ be a field. A non-zero $K$-derivation $d$ on $K\left[x_{1}, \ldots, x_{n}\right]$ is called integrable if there exist commuting $K$-derivations $\delta_{1}, \ldots, \delta_{n-1}$ on $K\left[x_{1}, \ldots, x_{n}\right]$ that are linearly independent from $d$ over $K\left(x_{1}, \ldots, x_{n}\right)$, and commute with $d$, that is, for all $a \in K\left[x_{1}, \ldots, x_{n}\right]$ and $i, j, 1 \leqslant i, j \leqslant$ $n-1$,

$$
d\left(\delta_{i}(a)\right)=\delta_{i}(d(a)) \quad \text { and } \quad \delta_{i}\left(\delta_{j}(a)\right)=\delta_{j}\left(\delta_{i}(a)\right)
$$

The following is a classical result.

Theorem 2.1. Let $d$ and $\delta$ be $\mathbb{R}$-derivations on $\mathbb{R}(x, y)$ defined by

$$
d(x)=f_{1}(x, y), d(y)=f_{2}(x, y), \delta(x)=g_{1}(x, y), \delta(y)=g_{2}(x, y) .
$$

Let $\left(x_{0}, y_{0}\right) \in \mathbb{R}^{2}$. Suppose that $d$ and $\delta$ commute and there is no $\left(\lambda_{1}, \lambda_{2}\right) \in \mathbb{R}^{2} \backslash\{(0,0)\}$ such that

$$
\lambda_{1}\left(\begin{array}{l}
f_{1}\left(x_{0}, y_{0}\right) \\
f_{2}\left(x_{0}, y_{0}\right)
\end{array}\right)=\lambda_{2}\left(\begin{array}{l}
g_{1}\left(x_{0}, y_{0}\right) \\
g_{2}\left(x_{0}, y_{0}\right)
\end{array}\right) .
$$

Then the initial value problem

$$
\dot{x}=f_{1}(x, y), \dot{y}=f_{2}(x, y), x(0)=x_{0}, y(0)=y_{0}
$$

has a solution given by

$$
(x(t), y(t))=F^{-1}(t, 0),
$$


where

$$
F\left(\begin{array}{l}
x \\
y
\end{array}\right)=\left(\begin{array}{l}
\int_{x_{0}}^{x} \frac{g_{2}(r, y)}{\Delta(r, y)} d r+\int_{y_{0}}^{y} \frac{-g_{1}\left(x_{0}, s\right)}{\Delta\left(x_{0}, s\right)} d s \\
\int_{x_{0}}^{x} \frac{-f_{2}(r, y)}{\Delta(r, y)} d r+\int_{y_{0}}^{y} \frac{f_{1}\left(x_{0}, s\right)}{\Delta\left(x_{0}, s\right)} d s
\end{array}\right),
$$

and $\Delta(x, y)=f_{1}(x, y) g_{2}(x, y)-f_{2}(x, y) g_{1}(x, y)$.

Example 2.1. Consider the initial value problem

$$
\dot{x}=1+x^{2}, \quad \dot{y}=-2 x y, \quad x(0)=x_{0}, \quad y(0)=y_{0},
$$

where $x_{0}$ and $y_{0}$ are real numbers and $y_{0} \neq 0$. The corresponding derivation is

$$
d(x)=1+x^{2}, \quad d(y)=-2 x y,
$$

and we observe that the derivation

$$
\delta(x)=0, \quad \delta(y)=y
$$

commutes with $d$, and that $d$ and $\delta$ are independent at $\left(x_{0}, y_{0}\right)$. Using the above formula, we obtain the solution

$$
x(t)=\tan \left(t+\tan ^{-1} x_{0}\right), \quad y(t)=y_{0}\left(1+x_{0}^{2}\right) \cos ^{2}\left(t+\tan ^{-1} x_{0}\right) .
$$

We make some observations, in the form of the following propositions:

Proposition 2.1. Let $K$ be a field. Every non-zero $K$-derivation of degree less than or equal to 1 on $K[x, y]$ is integrable.

Proof. We will consider the following cases. The symbols $a, b, c, e, f$, and $g$ are taken to be elements of $K$.

Case $0: d(x)=c, \quad d(y)=g$. Observe that $d$ commutes with any constant derivation.

Case $1: d(x)=a x, \quad d(y)=a y, \quad a \neq 0$. Observe that $d$ commutes with $\delta$, where $\delta(x)=y$, $\delta(y)=x$.

Case $2: d(x)=a x+b y, \quad d(y)=e x+f y, \quad$ different from Case 1. Observe that $d$ commutes with $\delta$, where $\delta(x)=x, \quad \delta(y)=y$.

Case $3: d(x)=a x+b y+c, \quad d(y)=e x+f y+g, \quad a f-b e \neq 0$. In this case, $d$ is equivalent to a derivation from Case 1 or Case 2 via a linear change of coordinates. Let $\left(x_{0}, y_{0}\right)$ be the solution to the system $a x+b y+c=e x+f y+g=0$. Now let $u=x-x_{0}$ and $v=y-y_{0}$, so that $d(u)=a u+b v$ and $d(v)=e u+f v$.

Case $4: d(x)=a x+b y+c, \quad d(y)=e x+f y+g, \quad a f-b e=0$

(a) $a=b=0$, different from Case 0 . If $e \neq 0$, then $d$ commutes with and is transversal to $\delta$ given by $\delta(x)=-\frac{g}{e}, \delta(y)=0$. If $f \neq 0$, then $d$ commutes with and is transversal to $\delta$ given by $\delta(x)=0, \delta(y)=-\frac{g}{f}$.

(b) at least one of $a$ and $b$ is not 0 . First assume $a \neq 0$. If $f=e=0$, then this is equivalent to Case 4 a by swapping the roles of $x$ and $y$. Assume at least one of $f$ and $e$ is not 0 . By the condition $a f-b e=0$, it must be that $e \neq 0$. Using the coordinate $z=e x-a y$ instead of $x$ puts this into the form of Case 4a. Next, assume $b \neq 0$. If $f=e=0$, then this is equivalent to Case $4 \mathrm{a}$. Assume at least one of $f$ and $e$ is not 0 . By the condition $a f-b e=0$, it must be that $f \neq 0$. Using the coordinate $z=f x-b y$ instead of $x$ puts this into the form of Case $4 \mathrm{a}$. 
Definition 2.3. Let $K$ be a field and let $d$ be a $K$-derivation on $K\left[x_{1}, \ldots, x_{n}\right]$. We say $d$ is divergencefree if

$$
\sum_{i=1}^{n} \frac{\partial}{\partial x_{i}} d\left(x_{i}\right)=0 .
$$

Proposition 2.2. Let $K$ be a field of characteristic 0 . There exist integrable divergence-free $K$ derivations on $K[x, y]$ that are not coordinate-change equivalent to a derivation of degree less than or equal to 1 .

Proof. The $K$-derivation defined by the same equations as $d$ from Example 2.1 is divergence-free and integrable. Note that the vector field corresponding to $d$ vanishes only at the points $(\sqrt{-1}, 0)$ and $(-\sqrt{-1}, 0)$ in $\bar{K}^{2}$. Since char $K=0$, these points are distinct. After a coordinate change, the number of points in $\bar{K}^{2}$ at which a vector field vanishes does not change. The vector field of any derivation of degree less than or equal to 1 vanishes at zero, one, or infinitely many points. We conclude that $d$ is not coordinate-change equivalent to a derivation of degree no greater than 1 .

In the following section, we study a class of divergence-free vector fields. We show that no member of this class is integrable.

\section{MAIN RESULT}

Fix a field $K$ of characteristic 0 . Suppose $\delta_{f}$ represents a second-order differential equation of the form

$$
\ddot{x}=f
$$

where $f \in K[x] \backslash K$, which corresponds to a conservative Newton system. That is,

$$
\delta_{f}\left(\begin{array}{l}
x \\
y
\end{array}\right)=\left(\begin{array}{l}
y \\
f
\end{array}\right)
$$

If $\operatorname{deg} f=1$, then $\delta_{f}$ is integrable by Proposition 2.1. The following theorem, which is our main result, addresses the case of $\operatorname{deg} f \geqslant 2$.

Theorem 3.1. For every

- $f \in K[x]$ such that $\operatorname{deg} f \geqslant 2$ and

- $K$-derivation $\gamma$ on $K[x, y]$ that commutes with $\delta_{f}$, where $\delta_{f}$ the $K$-derivation defined by (2), there exists $q \in K[H]$ such that

$$
\gamma=q \cdot \delta_{f}
$$

where $H=y^{2}-2 \int f d x$ and $\int f d x$ has 0 as the constant term.

As a corollary, we recover the following result on conservative Newton systems with a center at the origin. This result was first proven in [1, Theorem 11] and was given new proofs in [2, Theorem 4.1] and [4, Corollary 2.6] (see also [13, p. 30]).

Corollary 3.1. The real system

$$
\begin{aligned}
& \dot{x}=-y \\
& \dot{y}=f(x),
\end{aligned}
$$

with $f(0)=0, f^{\prime}(0)=1$, has a transversal commuting polynomial derivation if and only if $f(x)=$ $x$. 
Proof of Theorem 3.1. Fix $f \in K[x]$ such that $\operatorname{deg} f \geqslant 2$. Fix a $K$-derivation $\delta$ so that $\delta(x)=y$ and $\delta(y)=f$. Fix a $K$-derivation $\gamma$ such that $[\delta, \gamma]=0$. First consider the case in which $\operatorname{deg}_{y} \gamma \leqslant 1$.

Lemma 3.1. If

$$
\gamma\left(\begin{array}{l}
x \\
y
\end{array}\right)=\left(\begin{array}{l}
c_{1} y+c_{0} \\
d_{1} y+d_{0}
\end{array}\right)
$$

where $c_{1}, c_{0}, d_{1}, d_{0} \in K[x]$, and $[\delta, \gamma]=0$, then

$$
\gamma\left(\begin{array}{l}
x \\
y
\end{array}\right)=c_{1} \delta
$$

Proof. The equations $\delta(\gamma(x))=\gamma(\delta(x))$ and $\delta(\gamma(y))=\gamma(\delta(y))$ yield

$$
\left\{\begin{array}{l}
c_{1}^{\prime} y^{2}+c_{0}^{\prime} y+f c_{1}=d_{1} y+d_{0} \\
d_{1}^{\prime} y^{2}+d_{0}^{\prime} y+f d_{1}=f^{\prime} c_{1} y+f^{\prime} c_{0} .
\end{array}\right.
$$

Equating coefficients of like powers of $y$, we obtain the two independent systems

$$
c_{1}^{\prime}=0, \quad d_{0}^{\prime}=c_{1} f^{\prime}, \quad f c_{1}=d_{0}
$$

and

$$
d_{1}^{\prime}=0, \quad c_{0}^{\prime}=d_{1}, \quad f d_{1}=c_{0} f^{\prime} .
$$

The solution set of (3) is $c_{1}=$ constant, $d_{0}=c_{1} f$. System (4) has no non-zero solution, which we deduce as follows. We have

$$
\left(\frac{c_{0}}{f}\right)^{\prime}=\frac{c_{0}^{\prime} f-f^{\prime} c_{0}}{f^{2}}=0,
$$

so $c_{0}=($ const $) f$. Therefore, $d_{1}=($ const $) f^{\prime}$, which implies $d_{1}^{\prime}=($ const $) f^{\prime \prime}=0$. Since we assume $\operatorname{deg} f \geqslant 2$, the constant must be 0 . Therefore,

$$
\gamma\left(\begin{array}{l}
x \\
y
\end{array}\right)=c_{1}\left(\begin{array}{l}
y \\
f
\end{array}\right) .
$$

Now assume $\operatorname{deg}_{y} \gamma=M \geqslant 2$. Write

$$
\gamma\left(\begin{array}{l}
x \\
y
\end{array}\right)=\left(\begin{array}{c}
c_{M} y^{M}+\ldots+c_{0} \\
d_{M} y^{M}+\ldots+d_{0}
\end{array}\right),
$$

where for all $i, c_{i}, d_{i} \in K[x]$. Since $M=\operatorname{deg}_{y} \gamma$, at least one of $c_{M}$ and $d_{M}$ is non-zero. Now the system

$$
\left(\begin{array}{l}
\delta(\gamma(x)) \\
\delta(\gamma(y))
\end{array}\right)=\left(\begin{array}{l}
\gamma(\delta(x)) \\
\gamma(\delta(y))
\end{array}\right)
$$

becomes

$$
\begin{aligned}
\left(\begin{array}{c}
c_{M}^{\prime} y^{M+1}+c_{M-1}^{\prime} y^{M}+\ldots+c_{0}^{\prime} y \\
d_{M}^{\prime} y^{M+1}+d_{M-1}^{\prime} y^{M}+\ldots+d_{0}^{\prime} y
\end{array}\right)+\left(\begin{array}{c}
M f c_{M} y^{M-1}+\ldots+f c_{1} \\
M f d_{M} y^{M-1}+\ldots+f d_{1}
\end{array}\right) & =\left(\begin{array}{ll}
0 & 1 \\
f^{\prime} & 0
\end{array}\right)\left(\begin{array}{l}
c_{M} y^{M}+\ldots+c_{0} \\
d_{M} y^{M}+\ldots+d_{0}
\end{array}\right)
\end{aligned}
$$

Viewing these matrix entries as polynomials in $y$ and equating coefficients yields the following system of first-order ODEs 


$$
\begin{array}{cc}
c_{M}^{\prime}=0 & d_{M}^{\prime}=0 \\
c_{M-1}^{\prime}=d_{M} & d_{M-1}^{\prime}=f^{\prime} c_{M} \\
c_{M-2}^{\prime}+M f c_{M}=d_{M-1} & d_{M-2}^{\prime}+M f d_{M}=f^{\prime} c_{M-1} \\
c_{M-3}^{\prime}+(M-1) f c_{M-1}=d_{M-2} & d_{M-3}^{\prime}+(M-1) f d_{M-1}=f^{\prime} c_{M-2} \\
c_{M-4}^{\prime}+(M-2) f c_{M-2}=d_{M-3} & d_{M-4}^{\prime}+(M-2) f d_{M-2}=f^{\prime} c_{M-3} \\
c_{M-5}^{\prime}+(M-3) f c_{M-3}=d_{M-4} & d_{M-5}^{\prime}+(M-3) f d_{M-3}=f^{\prime} c_{M-4} \\
\vdots & \vdots \\
c_{0}^{\prime}+2 f c_{2}=d_{1} & d_{0}^{\prime}+2 f d_{2}=f^{\prime} c_{1} \\
f c_{1}=d_{0} & f d_{1}=f^{\prime} c_{0}
\end{array}
$$

as well as the condition

$$
c_{M} \neq 0 \text { or } d_{M} \neq 0 \text {. }
$$

In each equation, it is the case that if $c_{i}$ and $d_{j}$ both appear, then $i$ and $j$ have opposite parities. Thus, we see that this system consists of two independent systems. If $M$ is odd, these systems are:

$$
\begin{array}{cc}
(I o)_{M} & (I I o)_{M} \\
c_{M}^{\prime}=0 & d_{M}^{\prime}=0 \\
d_{M-1}^{\prime}=f^{\prime} c_{M} & c_{M-1}^{\prime}=d_{M} \\
c_{M-2}^{\prime}+M f c_{M}=d_{M-1} & d_{M-2}^{\prime}+M f d_{M}=f^{\prime} c_{M-1} \\
d_{M-3}^{\prime}+(M-1) f d_{M-1}=f^{\prime} c_{M-2} & c_{M-3}^{\prime}+(M-1) f c_{M-1}=d_{M-2} \\
c_{M-4}^{\prime}+(M-2) f c_{M-2}=d_{M-3} & d_{M-4}^{\prime}+(M-2) f d_{M-2}=f^{\prime} c_{M-3} \\
d_{M-5}^{\prime}+(M-3) f d_{M-3}=f^{\prime} c_{M-4} & c_{M-5}^{\prime}+(M-3) f c_{M-3}=d_{M-4} \\
\vdots & \vdots \\
c_{1}^{\prime}+3 f c_{3}=d_{2} & d_{1}^{\prime}+3 f d_{3}=f^{\prime} c_{2} \\
d_{0}^{\prime}+2 f d_{2}=f^{\prime} c_{1} & c_{0}^{\prime}+2 f c_{2}=d_{1} \\
f c_{1}=d_{0} & f d_{1}=f^{\prime} c_{0}
\end{array}
$$

If $M$ is even, the systems are:

$$
\begin{array}{cc}
(\text { IIe })_{M} & (\text { Ie })_{M} \\
c_{M}^{\prime}=0 & d_{M}^{\prime}=0 \\
d_{M-1}^{\prime}=f^{\prime} c_{M} & c_{M-1}^{\prime}=d_{M} \\
c_{M-2}^{\prime}+M f c_{M}=d_{M-1} & d_{M-2}^{\prime}+M f d_{M}=f^{\prime} c_{M-1} \\
d_{M-3}^{\prime}+(M-1) f d_{M-1}=f^{\prime} c_{M-2} & c_{M-3}^{\prime}+(M-1) f c_{M-1}=d_{M-2} \\
c_{M-4}^{\prime}+(M-2) f c_{M-2}=d_{M-3} & d_{M-4}^{\prime}+(M-2) f d_{M-2}=f^{\prime} c_{M-3} \\
d_{M-5}^{\prime}+(M-3) f d_{M-3}=f^{\prime} c_{M-4} & c_{M-5}^{\prime}+(M-3) f c_{M-3}=d_{M-4} \\
\vdots & \vdots \\
c_{0}^{\prime}+2 f c_{2}=d_{1} & d_{0}^{\prime}+2 f d_{2}=f^{\prime} c_{1} \\
f d_{1}=f^{\prime} c_{0} & f c_{1}=d_{0}
\end{array}
$$

In light of these observations, let

$$
\begin{aligned}
& n=\max \left\{i \mid i \text { odd and } c_{i} \neq 0 \text { or } i \text { even and } d_{i} \neq 0\right\}, \\
& p=\max \left\{i \mid i \text { even and } c_{i} \neq 0 \text { or } i \text { odd and } d_{i} \neq 0\right\} .
\end{aligned}
$$

Note that $n$ or $p$ may be undefined. Now write $\gamma=\gamma_{1}+\gamma_{2}$, where $\gamma_{1}(x)$ contains the terms of $\gamma(x)$ of odd degree in $y, \gamma_{1}(y)$ contains the terms of $\gamma(y)$ of even degree in $y, \gamma_{2}(x)$ contains the terms of 
$\gamma(x)$ of even degree in $y$, and $\gamma_{2}(y)$ contains the terms of $\gamma(y)$ of odd degree in $y$. Explicitly,

$$
\gamma_{1}\left(\begin{array}{l}
x \\
y
\end{array}\right)= \begin{cases}\left(\begin{array}{c}
c_{n} y^{n}+c_{n-2} y^{n-2}+\ldots+c_{1} y \\
d_{n-1} y^{n-1}+d_{n-3} y^{n-3}+\ldots+d_{0}
\end{array}\right) & \text { if } n \text { is odd, } \\
\left(\begin{array}{c}
c_{n-1} y^{n-1}+c_{n-3} y^{n-3}+\ldots+c_{1} y \\
d_{n} y^{n}+d_{n-2} y^{n-2}+\ldots+d_{0}
\end{array}\right) & \text { if } n \text { is even, } \\
\left(\begin{array}{l}
0 \\
0
\end{array}\right) & \text { if } n \text { is undefined, }\end{cases}
$$

and

$$
\gamma_{2}\left(\begin{array}{l}
x \\
y
\end{array}\right)= \begin{cases}\left(\begin{array}{c}
c_{p-1} y^{p-1}+c_{p-3} y^{p-3}+\ldots+c_{0} \\
d_{p} y^{p}+d_{p-2} y^{p-2}+\ldots+d_{1} y
\end{array}\right) & \text { if } p \text { is odd }, \\
\left(\begin{array}{c}
c_{p} y^{p}+c_{p-2} y^{p-2}+\ldots+c_{0} \\
d_{p-1} y^{p-1}+d_{p-3} y^{p-3}+\ldots+d_{1} y
\end{array}\right) & \text { if } p \text { is even, } \\
\left(\begin{array}{l}
0 \\
0
\end{array}\right) & \text { if } p \text { is undefined. }\end{cases}
$$

As we have seen, the criterion $[\delta, \gamma]=0$ is equivalent to the conjunction of two systems of equations in which one system only involves the terms of $\gamma_{1}$ and the other only involves the terms of $\gamma_{2}$. Hence, $\left[\delta, \gamma_{1}\right]=\left[\delta, \gamma_{2}\right]=0$.

Let us examine the possible values of $n$. If $n$ is undefined, then $\gamma_{1}(x, y)=(0,0)$. If $n=0$, then $\gamma_{1}$ is the same as the $\gamma$ of Lemma 3.1 with $c_{1}=c_{0}=d_{1}=0$. Thus, by Lemma 3.1, $\gamma_{1}=0$, which contradicts that $n=0$. If $n=1$, then $\gamma_{1}$ is the same as the $\gamma$ of Lemma 3.1 with $c_{0}=d_{1}=0$. Thus by Lemma 3.1, $\gamma_{1}=c_{1} \delta$, and, in the proof of Lemma 3.1, it is shown that $c_{1} \in K$. If $n \geqslant 2$ is even, the coefficients of $\gamma_{1}$ must satisfy $(I e)_{n}$ and $d_{n} \neq 0$. We will show in Lemma 3.4 and Corollary 3.2 that this is impossible. If $n$ is odd, the coefficients of $\gamma_{1}$ must satisfy $(I o)_{n}$ and $c_{n} \neq 0$. We will show in Lemma 3.2 and Lemma 3.3 that this implies $\gamma_{1}=q \delta$ for some $q \in K[H]$. In summary,

- If $n$ is undefined, then $\gamma_{1}=0 \cdot \delta$.

- It is impossible that $n=0$.

- If $n=1$, then $\gamma_{1}=c_{1} \cdot \delta$ and $c_{1} \in K$.

- It is impossible that $n \geqslant 2$ is even. (Lemma 3.4, Corollary 3.2)

- If $n \geqslant 3$ is odd, then $\gamma_{1}=q \cdot \delta$ for some $q \in K[H]$. (Lemmas 3.2, 3.3)

Let us examine the possible values of $p$. If $p$ is undefined, then $\gamma_{2}(x, y)=(0,0)$. If $p=0$, then $\gamma_{2}$ is the same as the $\gamma$ from Lemma 3.1 with $c_{1}=d_{1}=d_{0}=0$. Thus, by Lemma 3.1, $\gamma_{2}=0$, which contradicts that $p=0$. If $p=1$, then $\gamma_{2}$ is the same as the $\gamma$ of Lemma 3.1 with $c_{1}=d_{0}=0$. Thus, by Lemma 3.1, $\gamma_{2}=0$, which contradicts that $p=1$. If $p \geqslant 2$ is even, the coefficients of $\gamma_{2}$ must satisfy $(I I e)_{p}$ and $c_{p} \neq 0$. We will show in Lemma 3.5 and Corollary 3.3 that this is impossible. If $p \geqslant 3$ is odd, the coefficients of $\gamma_{2}$ must satisfy $(I I o)_{p}$ and $d_{p} \neq 0$. We will show in Lemma 3.6, Lemma 3.7, Lemma 3.9, and Corollary 3.4 that this is impossible. We summarize these results as follows: 
- If $p$ is undefined, then $\gamma_{2}=0 \cdot \delta$.

- It is impossible that $p=0$.

- It is impossible that $p=1$.

- It is impossible that $p \geqslant 2$ is even. (Lemma 3.5, Corollary 3.3)

- It is impossible that $p \geqslant 3$ is odd. (Lemmas 3.6, 3.7, 3.9, Corollary 3.4)

From the bulleted statements, it follows that $\gamma_{1}=q \delta$ for some $q \in K[H]$ and $\gamma_{2}=0$. These lemmas and their corollaries constitute the rest of the proof of Theorem 3.1.

Definition 3.1. Let $a \in K[x, y]$. We define $\int a d x$ to be the element of $K[x, y]$ whose partial derivative with respect to $x$ is $a$ and whose constant term is 0 .

Lemma 3.2. For every odd integer $m \geqslant 3$, the solution set of $(\text { Io })_{m}$, with $c_{0}, \ldots, d_{m}$ treated as variables, is an $\frac{m+1}{2}$-dimensional $K$-vector space.

Proof. Fix $m \geqslant 3$. Label the equations of $(I o)_{m}$ as follows:

$$
\begin{array}{cc}
e_{m+1} & c_{m}^{\prime}=0 \\
e_{m} & d_{m-1}^{\prime}=f^{\prime} c_{m} \\
e_{m-1} & c_{m-2}^{\prime}+m f c_{m}=d_{m-1} \\
\vdots & \vdots \\
e_{1} & d_{0}^{\prime}+2 f d_{2}=f^{\prime} c_{1} \\
e_{0} & f c_{1}=d_{0}
\end{array}
$$

We show the following by induction on $k, 0 \leqslant k \leqslant \frac{m-3}{2}$ :

The solution set of $\left\{e_{m+1}, e_{m}, \ldots, e_{m-2 k-2}, d_{m-2 k-3}=f c_{m-2 k-2}\right\}$ is a $K$-vector space of dimension $k+2$.

Base Case: $k=0$

The system

$$
\left\{e_{m+1}, e_{m}, e_{m-1}, e_{m-2}, d_{m-3}=f c_{m-2}\right\}
$$

is

$$
\begin{array}{ll}
e_{m+1}: & c_{m}^{\prime}=0 \\
e_{m}: & d_{m-1}^{\prime}=f^{\prime} c_{m} \\
e_{m-1}: & c_{m-2}^{\prime}=-m f c_{m}+d_{m-1} \\
e_{m-2}: & d_{m-3}^{\prime}=-(m-1) f d_{m-1}+f^{\prime} c_{m-2} \\
& d_{m-3}=f c_{m-2}
\end{array}
$$

Let $\left(\tilde{d}_{m-3}, \tilde{c}_{m-2}, \tilde{d}_{m-1}, \tilde{c}_{m}\right)$ be a solution of (8). By $e_{m+1}, \tilde{c}_{m}=a_{1}$ for some $a_{1} \in K$. It follows that

$$
f^{\prime} \tilde{c}_{m-2}+f \tilde{c}_{m-2}^{\prime}=-(m-1) f \tilde{d}_{m-1}+f^{\prime} \tilde{c}_{m-2},
$$

and hence

$$
\tilde{c}_{m-2}^{\prime}=-(m-1) \tilde{d}_{m-1}
$$

and so

$$
\tilde{d}_{m-1}=m f \tilde{c}_{m}+\tilde{c}_{m-2}^{\prime}=m f \tilde{c}_{m}-(m-1) \tilde{d}_{m-1}
$$

Thus

$$
\tilde{d}_{m-1}=f \tilde{c}_{m}=a_{1} f .
$$


It follows from this and $e_{m-1}$ that

$$
\tilde{c}_{m-2}^{\prime}=-(m-1) a_{1} f
$$

and hence

$$
\tilde{c}_{m-2}=-\int(m-1) a_{1} f d x+a_{2}
$$

for some $a_{2} \in K$. From this and the condition $\tilde{d}_{m-3}=f \tilde{c}_{m-2}$ it follows that

$$
\tilde{d}_{m-3}=f\left(-\int(m-1) a_{1} f d x+a_{2}\right) \text {. }
$$

One can verify that

$$
\left(f\left(-\int(m-1) a_{1} f d x+a_{2}\right), \quad-\int(m-1) a_{1} f d x+a_{2}, \quad a_{1} f, \quad a_{1}\right)
$$

is indeed a solution of (8). We have just shown that the solution set of (8) is exactly the elements of $K[x]^{4}$ of the form (9) with $a_{1}, a_{2} \in K$. This set is the $K$-span of the tuples

$$
\left(f\left(-\int(m-1) f d x\right),-\int(m-1) f d x, f, 1\right) \text { and }(f, 1,0,0) .
$$

Hence, the solution space is a two-dimensional $K$-vector space.

Inductive Step: Fix $k, 0 \leqslant k<\frac{m-3}{2}$. Consider

$$
\begin{aligned}
& \left\{e_{m+1}, e_{m}, \ldots, e_{m-2 k-2}, d_{m-2 k-3}=f c_{m-2 k-2}\right\} \\
& \left\{e_{m+1}, e_{m}, \ldots, e_{m-2 k-4}, d_{m-2 k-5}=f c_{m-2 k-4}\right\}
\end{aligned}
$$

Assume

(12) The solution set of (10) is a $K$-vector space of dimension $k+2$.

We will show

(13) The solution set of (11) is a $K$-vector space of dimension $k+3$.

We first show that

(14) The solution set of (11) is the solution set of

$$
\left\{e_{m+1}, \ldots, e_{m-2 k-2}, e_{m-2 k-3}, d_{m-2 k-3}=f c_{m-2 k-2}, d_{m-2 k-5}=f c_{m-2 k-4}\right\} .
$$

For ease of reference, we write the equations $e_{m-2 k-3}$ and $e_{m-2 k-4}$ :

$$
\begin{array}{ll}
e_{m-2 k-3}: & c_{m-2 k-4}^{\prime}=-(m-2 k-2) f c_{m-2 k-2}+d_{m-2 k-3} \\
e_{m-2 k-4}: & d_{m-2 k-5}^{\prime}=-(m-2 k-3) f d_{m-2 k-3}+f^{\prime} c_{m-2 k-4}
\end{array}
$$

Suppose $\left(\tilde{d}_{m-2 k-5}, \ldots, \tilde{c}_{m}\right)$ is a solution of

$$
\left\{e_{m+1}, \ldots, e_{m-2 k-4}, d_{m-2 k-5}=f c_{m-2 k-4}\right\} \text {. }
$$

Then $\left(\tilde{d}_{m-2 k-3}, \ldots, \tilde{c}_{m}\right)$ is a solution of $\left\{e_{m+1}, \ldots, e_{m-2 k-2}\right\}$. We now show that

$$
\tilde{d}_{m-2 k-3}=f \tilde{c}_{m-2 k-2} \text {. }
$$

Since $\left(\tilde{d}_{m-2 k-5}, \ldots, \tilde{c}_{m}\right)$ satisfies $e_{m-2 k-4}$, we have

$$
\tilde{d}_{m-2 k-5}^{\prime}=-(m-2 k-3) f \tilde{d}_{m-2 k-3}+f^{\prime} \tilde{c}_{m-2 k-4} .
$$


Since $\tilde{d}_{m-2 k-5}=f \tilde{c}_{m-2 k-4}$, it follows that

$$
\tilde{d}_{m-2 k-5}^{\prime}=f^{\prime} \tilde{c}_{m-2 k-4}+f \tilde{c}_{m-2 k-4}^{\prime} .
$$

Combining this with (17), we get

$$
f \tilde{c}_{m-2 k-4}^{\prime}=-(m-2 k-3) f \tilde{d}_{m-2 k-3},
$$

and hence

$$
\tilde{c}_{m-2 k-4}^{\prime}=-(m-2 k-3) \tilde{d}_{m-2 k-3}
$$

Since $\left(\tilde{d}_{m-2 k-5}, \ldots, \tilde{c}_{m}\right)$ satisfies $e_{m-2 k-3}$, we have

$$
\tilde{c}_{m-2 k-4}^{\prime}+(m-2 k-2) f \tilde{c}_{m-2 k-2}=\tilde{d}_{m-2 k-3},
$$

and combining this with (18) gives us (16).

We now show the opposite inclusion. Suppose $\left(\tilde{d}_{m-2 k-5}, \ldots, \tilde{c}_{m}\right)$ satisfies (15). Since the tuple satisfies $d_{m-2 k-5}=f c_{m-2 k-4}, e_{m-2 k-3}$, and $d_{m-2 k-3}=f c_{m-2 k-2}$, we have

$$
\begin{aligned}
\tilde{d}_{m-2 k-5}^{\prime} & =f^{\prime} \tilde{c}_{m-2 k-4}+f \tilde{c}_{m-2 k-4}^{\prime} \\
& =f^{\prime} \tilde{c}_{m-2 k-4}+f\left(-(m-2 k-2) f \tilde{c}_{m-2 k-2}+\tilde{d}_{m-2 k-3}\right) \\
& =f^{\prime} \tilde{c}_{m-2 k-4}+f\left(-(m-2 k-2) \tilde{d}_{m-2 k-3}+\tilde{d}_{m-2 k-3}\right) \\
& =f^{\prime} \tilde{c}_{m-2 k-4}-(m-2 k-3) f \tilde{d}_{m-2 k-3} .
\end{aligned}
$$

Thus the tuple also satisfies $e_{m-2 k-4}$. This completes the proof of (14).

Now we show (13). Since (11) is a system consisting of homogeneous linear differential equations and a homogeneous linear equation in $2 k+6$ variables, the solution set is a $K$-vector subspace of $K[x]^{2 k+6}$. Let $W$ denote this vector space, let $\pi_{i}: K[x]^{2 k+6} \rightarrow K[x]$ be projection onto the $i$-th coordinate, and let $\pi: K[x]^{2 k+6} \rightarrow K[x]^{2 k+4}$ be the projection onto the last $2 k+4$ coordinates. Similarly, the solution set of (10) is a $K$-vector subspace of $K[x]^{2 k+4}$. Call this space $V$. By (12), $\operatorname{dim} V=k+2$. Let $p_{i}: K[x]^{2 k+4} \rightarrow K[x]$ be the projection onto the $i$-th coordinate.

Let $a_{1}, \ldots, a_{k+2} \in K[x]^{2 k+4}$ be a basis for $V$. For each $i=1, \ldots, k+2$, we define $b_{i} \in K[x]^{2 k+6}$ as follows. Let

$$
\pi\left(b_{i}\right)=a_{i}, \quad \pi_{2}\left(b_{i}\right)=\int\left(-(m-2 k-2) f p_{2}\left(a_{i}\right)+p_{1}\left(a_{i}\right)\right) d x, \quad \pi_{1}\left(b_{i}\right)=f \pi_{2}\left(b_{i}\right) .
$$

By (14), each $b_{i}$ is a solution of (11). Since $d_{m-2 k-5}$ and $c_{m-2 k-4}$ only appear in the equations

$$
\begin{gathered}
c_{m-2 k-4}^{\prime}+(m-2 k-2) f c_{m-2 k-2}=d_{m-2 k-3}, \\
d_{m-2 k-5}^{\prime}+(m-2 k-3) f d_{m-2 k-3}=f^{\prime} c_{m-2 k-4}, \\
d_{m-2 k-5}=f c_{m-2 k-4}
\end{gathered}
$$

of (11), we observe that

$$
b_{k+3}:=(f, 1,0, \ldots, 0) \in W .
$$

We show that

$$
\operatorname{span}_{K}\left\{b_{1}, \ldots, b_{k+3}\right\}=W .
$$

Suppose $w \in W$. By (14), $\pi(w) \in V$, so there exist $\alpha_{i} \in K, 1 \leqslant i \leqslant k+2$, such that

$$
\pi(w)=\sum_{i=1}^{k+2} \alpha_{i} \pi\left(b_{i}\right) .
$$


Also by (14), there is a $\beta \in K$ such that

$$
\begin{aligned}
\pi_{2}(w) & =\int\left(-(m-2 k-2) f \pi_{4}(w)+\pi_{3}(w)\right) d x+\beta \\
& =\int\left(-(m-2 k-2) f \sum_{i=1}^{k+2} \alpha_{i} \pi_{4}\left(b_{i}\right)+\sum_{i=1}^{k+2} \alpha_{i} \pi_{3}\left(b_{i}\right)\right) d x+\beta \\
& =\sum_{i=1}^{k+2} \alpha_{i} \int\left(-(m-2 k-2) f \pi_{4}\left(b_{i}\right)+\pi_{3}\left(b_{i}\right)\right) d x+\beta=\sum_{i=1}^{k+2} \alpha_{i} \pi_{2}\left(b_{i}\right)+\beta .
\end{aligned}
$$

By (14), we have $\pi_{1}(w)=f \pi_{2}(w)$. Using the fact that $\pi_{1}\left(b_{i}\right)=f \pi_{2}\left(b_{i}\right)$, we get

$$
\pi_{1}(w)=\sum_{i=1}^{k+2} \alpha_{i} \pi_{1}\left(b_{i}\right)+f \beta .
$$

Thus,

$$
w=\sum_{i=1}^{k+2} \alpha_{i} b_{i}+\beta b_{k+3} .
$$

We conclude that $\operatorname{span}_{K}\left\{b_{1}, \ldots, b_{k+3}\right\}=W$.

Since $\left\{\pi\left(b_{1}\right), \ldots, \pi\left(b_{k+2}\right)\right\}$ is $K$-linearly independent, $\left\{b_{1}, \ldots, b_{k+2}\right\}$ is $K$-linearly independent. Since the constant term of $\pi_{2}\left(b_{i}\right)$ is 0 for $i=1, \ldots, k+2$, it is clear that

$$
b_{k+3} \notin \operatorname{span}_{K}\left\{b_{1}, \ldots, b_{k+2}\right\} .
$$

We conclude that $\operatorname{dim}_{K} W=k+3$. This completes the inductive step.

Setting $k=\frac{m-3}{2}$ in (7) proves the lemma.

Lemma 3.3. If $n \geqslant 3$ is odd, then $\gamma_{1}=q \delta$ for some $q \in K[H]$.

Proof. Recall that, if $n \geqslant 3$ is odd, the coefficients of $\gamma_{1}$ must satisfy $(I o)_{n}$. Observe that $\delta(H)=0$. Hence, any $K$-derivation $D$ of the form

$$
D\left(\begin{array}{l}
x \\
y
\end{array}\right)=\left(a_{\frac{n-1}{2}} H^{\frac{n-1}{2}}+a_{\frac{n-1}{2}-1} H^{\frac{n-1}{2}-1}+\ldots+a_{0}\right) \cdot\left(\begin{array}{l}
y \\
f
\end{array}\right), \quad a_{i} \in K,
$$

commutes with $\delta$. Writing $D$ in the form of (5), we see that $c_{i}=0$ for even $i$ and $d_{i}=0$ for odd $i$, so a choice of

$$
a_{0}, \ldots, a_{\frac{n-1}{2}}
$$

provides a solution to $(\mathrm{Io})_{n}$. Moreover, two distinct choices of $a_{0}, \ldots, a_{\frac{n-1}{2}}$ provide two distinct solutions of $(I o)_{n}$. Thus, the set of solutions of $(I o)_{n}$ that correspond to derivations of the form $q \delta$, where $q \in K[H]$, is a $K$-vector space of dimension $\frac{n+1}{2}$. Since this vector space is contained in the vector space of solutions to $(I o)_{n}$, which by Lemma 3.2 has dimension $\frac{n+1}{2}$, the spaces must be equal.

Lemma 3.4. For all even $m \geqslant 2$, the system $(\text { Ie })_{m}$ implies $d_{m}=0$.

Proof. Fix even $m \geqslant 2$. Label the equations in $(I e)_{m}$ as follows: 


$$
\begin{array}{ll}
e_{m+1}: & d_{m}^{\prime}=0 \\
e_{m}: & c_{m-1}^{\prime}=d_{m} \\
e_{m-1}: & d_{m-2}^{\prime}+m f d_{m}=f^{\prime} c_{m-1} \\
e_{m-2}: & c_{m-3}^{\prime}+(m-1) f c_{m-1}=d_{m-2} \\
& \vdots \\
e_{1}: & d_{0}^{\prime}+2 f d_{2}=f^{\prime} c_{1} \\
e_{0}: & f c_{1}=d_{0}
\end{array}
$$

We show by induction on $k, 0 \leqslant k \leqslant \frac{m-2}{2}$, that

$$
\left\{e_{0}, e_{1}, \ldots, e_{2 k+1}\right\} \quad \text { implies } \quad c_{2 k+1}^{\prime}=-(2 k+2) d_{2 k+2} \text {. }
$$

The case $k=0$ is straightforward. For the inductive hypothesis, fix $k, 0 \leqslant k<\frac{m-2}{2}$, and assume (19). Now assume $\left\{e_{0}, e_{1}, \ldots, e_{2 k+3}\right\}$. Equations $e_{2 k+2}$ and $e_{2 k+3}$ are

$$
c_{2 k+1}^{\prime}=-(2 k+3) f c_{2 k+3}+d_{2 k+2} \quad \text { and } \quad d_{2 k+2}^{\prime}=-(2 k+4) f d_{2 k+4}+f^{\prime} c_{2 k+3},
$$

and the inductive hypothesis gives us

$$
c_{2 k+1}^{\prime}=-(2 k+2) d_{k+2} .
$$

Equating the two expressions for $c_{2 k+1}^{\prime}$, we obtain $d_{2 k+2}=f c_{2 k+3}$. Differentiating this and equating the two expressions for $d_{2 k+2}^{\prime}$ gives us

$$
f^{\prime} c_{2 k+3}+f c_{2 k+3}^{\prime}=-(2 k+4) f d_{2 k+4}+f^{\prime} c_{2 k+3},
$$

which implies

$$
c_{2 k+3}^{\prime}=-(2 k+4) d_{2 k+4} .
$$

This completes the inductive step. This shows that a consequence of $(I e)_{m}$ is

$$
c_{m-1}^{\prime}=-m d_{m}
$$

Since $m$ was assumed to be even, we have $m \neq-1$. In order that $e_{m}$ and $c_{m-1}^{\prime}=-m d_{m}$ both be satisfied, it is necessary that $d_{m}=0$.

Corollary 3.2. It is impossible that $n$ is an even integer greater than or equal to 2.

Proof. Suppose $n \geqslant 2$ and $n$ is even. Then the coefficients of $\gamma_{1}$ must satisfy $(I e)_{n}$, and also $d_{n} \neq 0$. But by Lemma 3.4, $d_{n}=0$ is a consequence of $(I e)_{n}$.

Lemma 3.5. For all even $m \geqslant 2$, the system (IIe $)_{m}$ implies $c_{m}=0$.

Proof. Fix even $m \geqslant 2$. Label the equations of $(I I e)_{m}$ as follows:

$$
\begin{array}{ll}
e_{m+1}: & c_{m}^{\prime}=0 \\
e_{m}: & d_{m-1}^{\prime}=f^{\prime} c_{m} \\
e_{m-1}: & c_{m-2}^{\prime}+m f c_{m}=d_{m-1} \\
e_{m-2}: & d_{m-3}^{\prime}+(m-1) f d_{m-1}=f^{\prime} c_{m-2} \\
& \vdots \\
e_{1}: & c_{0}^{\prime}+2 f c_{2}=d_{1} \\
e_{0}: & f d_{1}=f^{\prime} c_{0}
\end{array}
$$


We first show the following by induction on $k, 0 \leqslant k \leqslant \frac{m-2}{2}$ :

$$
\begin{aligned}
& \text { If }\left(\tilde{d}_{m-2 k-1}, \ldots, \tilde{c}_{m}\right) \text { is a solution of }\left\{e_{m+1}, \ldots, e_{m-2 k}\right\} \text { with } \tilde{c}_{m} \neq 0, \\
& \text { then } \tilde{d}_{m-2 k-1} \neq 0, \operatorname{deg}\left(\tilde{d}_{m-2 k-1}\right)=\operatorname{deg}\left(f \cdot \tilde{c}_{m-2 k}\right), \text { and } \operatorname{lc}\left(\tilde{d}_{m-2 k-1}\right)=\operatorname{lc}\left(f \cdot \tilde{c}_{m-2 k}\right) .
\end{aligned}
$$

Base Case, $k=0$ :

Suppose $\left(\tilde{d}_{m-1}, \tilde{c}_{m}\right)$ is a solution of $\left\{c_{m}^{\prime}=0, d_{m-1}^{\prime}=f^{\prime} c_{m}\right\}$ and $c_{m} \neq 0$. Since $\operatorname{deg} f \geqslant 2$ and $\tilde{c}_{m}$ is a non-zero constant,

$$
\tilde{d}_{m-1} \neq 0 \quad \text { and } \quad \operatorname{deg} \tilde{d}_{m-1}=\operatorname{deg}\left(f \tilde{c}_{m}\right)=\operatorname{deg} f .
$$

We have $\operatorname{lc}\left(\tilde{d}_{m-1}^{\prime}\right)=\operatorname{deg} f \cdot \operatorname{lc} f \cdot \tilde{c}_{m}$. Since $\tilde{c}_{m}$ is a constant and $\operatorname{deg} \tilde{d}_{m-1}=\operatorname{deg} f$, we have

$$
\operatorname{lc}\left(\tilde{d}_{m-1}\right)=\operatorname{lc}\left(f \tilde{c}_{m}\right) \text {. }
$$

Inductive Step:

Fix $k, 0 \leqslant k<\frac{m-2}{2}$. Assume (20) for this $k$. Suppose $\left(\tilde{d}_{m-2 k-3}, \ldots, \tilde{c}_{m}\right)$ is a solution of $\left\{e_{m+1}, \ldots, e_{m-2 k-2}\right\}$ such that $\tilde{c}_{m} \neq 0$. For ease of reference, we write:

$$
\begin{array}{ll}
e_{m-2 k-1}: & c_{m-2 k-2}^{\prime}+(m-2 k) \cdot f \cdot c_{m-2 k}=d_{m-2 k-1} \\
e_{m-2 k-2}: & d_{m-2 k-3}^{\prime}+(m-2 k-1) \cdot f \cdot d_{m-2 k-1}=f^{\prime} \cdot c_{m-2 k-2}
\end{array}
$$

Then

$$
\tilde{c}_{m-2 k-2}^{\prime}=\tilde{d}_{m-2 k-1}-(m-2 k) f \cdot \tilde{c}_{m-2 k} .
$$

Since $m$ is even, $m-2 k-1 \neq 0$. Therefore, by the inductive hypothesis,

$$
\operatorname{deg}\left(\tilde{c}_{m-2 k-2}^{\prime}\right)=\operatorname{deg}\left(\tilde{d}_{m-2 k-1}\right) \geqslant 0
$$

and we have

$$
\operatorname{lc}\left(\tilde{c}_{m-2 k-2}^{\prime}\right)=-(m-2 k-1) \cdot \operatorname{lc}\left(\tilde{d}_{m-2 k-1}\right),
$$

and hence

$$
\operatorname{deg} \tilde{c}_{m-2 k-2} \cdot \operatorname{lc}\left(\tilde{c}_{m-2 k-2}\right)=-(m-2 k-1) \cdot \operatorname{lc}\left(\tilde{d}_{m-2 k-1}\right) .
$$

By equation $e_{m-2 k-2}$, we have

$$
\tilde{d}_{m-2 k-3}^{\prime}=f^{\prime} \cdot \tilde{c}_{m-2 k-2}-(m-2 k-1) \cdot f \cdot \tilde{d}_{m-2 k-1} .
$$

We will show that the degrees of the two terms on the right-hand side of (23) are equal and that their leading coefficients do not cancel. From (21), it follows that

$$
\operatorname{deg} \tilde{c}_{m-2 k-2}=\operatorname{deg} \tilde{d}_{m-2 k-1}+1,
$$

so that

$$
\operatorname{deg}\left(f^{\prime} \cdot \tilde{c}_{m-2 k-2}\right)=\operatorname{deg}\left(f \cdot \tilde{d}_{m-2 k-1}\right)
$$

Observe that

and, using (22),

$$
\operatorname{lc}\left(f^{\prime} \cdot \tilde{c}_{m-2 k-2}\right)=\operatorname{deg} f \cdot \operatorname{lc} f \cdot \operatorname{lc}\left(\tilde{c}_{m-2 k-2}\right)
$$

$$
\operatorname{lc}\left(f \cdot \tilde{d}_{m-2 k-1}\right)=\operatorname{lc} f \cdot \operatorname{lc}\left(\tilde{d}_{m-2 k-1}\right)=\operatorname{lc} f \cdot \frac{-1}{m-2 k-1} \cdot \operatorname{lc}\left(\tilde{c}_{m-2 k-2}\right) \cdot \operatorname{deg} \tilde{c}_{m-2 k-2} .
$$

It follows that

$$
\operatorname{lc}\left(f^{\prime} \cdot \tilde{c}_{m-2 k-2}\right) \neq(m-2 k-1) \cdot \operatorname{lc}\left(f \cdot \tilde{d}_{m-2 k-1}\right),
$$

and, together with (23) and (24), this gives us

$$
\operatorname{lc}\left(\tilde{d}_{m-2 k-3}^{\prime}\right)=\operatorname{lc} f \cdot \operatorname{lc}\left(\tilde{c}_{m-2 k-2}\right) \cdot\left(\operatorname{deg} f+\operatorname{deg} \tilde{c}_{m-2 k-2}\right) .
$$


By (23), (24), and (25), we have

$$
\operatorname{deg}\left(\tilde{d}_{m-2 k-3}\right)=\operatorname{deg} f+\operatorname{deg} \tilde{c}_{m-2 k-2}
$$

Combining (26) and (27) gives us

$$
\operatorname{lc}\left(\tilde{d}_{m-2 k-3}\right)=\operatorname{lc} f \cdot \operatorname{lc}\left(\tilde{c}_{m-2 k-2}\right) .
$$

This completes the inductive step.

We proceed with the proof of the lemma. Let $\left(\tilde{c}_{0}, \ldots, \tilde{c}_{m}\right)$ be a solution of $(I I e)_{m}$ with $\tilde{c}_{m} \neq 0$. We will derive a contradiction. It follows immediately that $\left(\tilde{d}_{1}, \ldots, \tilde{c}_{m}\right)$ is a solution of $\left\{e_{m+1}, \ldots, e_{1}\right\}$. Setting $k=\frac{m-2}{2}$ in (20), we have that $\operatorname{deg}\left(\tilde{d}_{1}\right)=\operatorname{deg}\left(f \cdot \tilde{c}_{2}\right) \geqslant 0$ and

$$
\operatorname{lc}\left(\tilde{d}_{1}\right)=\operatorname{lc}(f) \cdot \operatorname{lc}\left(\tilde{c}_{2}\right) \text {. }
$$

From $e_{0}$, we see that

$$
\operatorname{deg}\left(\tilde{d}_{1}\right)=\operatorname{deg}\left(\tilde{c}_{0}\right)-1=\operatorname{deg}\left(\tilde{c}_{0}^{\prime}\right)
$$

By equation $e_{1}$, we have

$$
\operatorname{lc}\left(\tilde{d}_{1}\right)=2 \cdot \operatorname{lc} f \cdot \operatorname{lc}\left(\tilde{c}_{2}\right)+\operatorname{deg} \tilde{c}_{0} \cdot \operatorname{lc}\left(\tilde{c}_{0}\right) .
$$

Therefore, by (28), we have

$$
\operatorname{lc} f \cdot \operatorname{lc}\left(\tilde{c}_{2}\right)=2 \cdot \operatorname{lc} f \cdot \operatorname{lc}\left(\tilde{c}_{2}\right)+\operatorname{deg} \tilde{c}_{0} \cdot \operatorname{lc}\left(\tilde{c}_{0}\right)
$$

and hence

By equation $e_{0}$, we have

$$
\operatorname{lc}\left(\tilde{c}_{0}\right)=\frac{-\operatorname{lc} f \cdot \operatorname{lc}\left(\tilde{c}_{2}\right)}{\operatorname{deg} \tilde{c}_{0}}
$$

$$
\operatorname{lc} f \cdot \operatorname{lc}\left(\tilde{d}_{1}\right)=\operatorname{deg} f \cdot \operatorname{lc} f \cdot \operatorname{lc}\left(\tilde{c}_{0}\right)=\operatorname{deg} f \cdot \operatorname{lc} f \cdot\left(\frac{-\operatorname{lc} f \cdot \operatorname{lc}\left(\tilde{c}_{2}\right)}{\operatorname{deg} \tilde{c}_{0}}\right) .
$$

By (28),

It follows that

$$
\operatorname{lc} f \cdot \operatorname{lc} f \cdot \operatorname{lc}\left(\tilde{c}_{2}\right)=\operatorname{deg} f \cdot \operatorname{lc} f \cdot\left(\frac{-\operatorname{lc} f \cdot \operatorname{lc}\left(\tilde{c}_{2}\right)}{\operatorname{deg} \tilde{c}_{0}}\right) \text {. }
$$

which is a contradiction, since $\operatorname{deg} f>0$.

Corollary 3.3. It is impossible that $p$ is an even integer greater than or equal to 2.

Proof. Suppose $p \geqslant 2$ and $p$ is even. Then the coefficients of $\gamma_{2}$ must satisfy $(I I e)_{p}$, together with $c_{p} \neq 0$. But by Lemma 3.5, $(\text { IIe })_{p}$ implies $c_{p}=0$.

In the lemmas that follow, we refer to $K$-derivations on the ring $K\left[x^{1 / t}, x^{-1 / t}, y\right]$, where $t$ is a positive integer. We view this ring as isomorphic to

$$
K[x, y, z, w] /\left(z^{t}-x, z w-1\right) .
$$

By [7, Lemma II.2.1], since char $K=0$, any $K$-derivation on $K[x, y]$ extends uniquely to a $K$ derivation on $K\left[x^{1 / t}, x^{-1 / t}, y\right]$. One consequence of this is that a $K$-derivation on $K\left[x^{1 / t}, x^{-1 / t}, y\right]$ can be defined by stating its action on $x$ and $y$.

Lemma 3.6. For every odd integer $m$ greater than or equal to 3 , there exists $P_{m}(X) \in \mathbb{Z}[X] \backslash\{0\}$ such that:

- $\operatorname{deg} P_{m} \leqslant \frac{m+1}{2}$ 
- for every

- positive integer $t$

- $h \in K\left[x^{1 / t}, x^{-1 / t}\right] \backslash\{0\}$,

if the $K$-derivation

$$
\beta\left(\begin{array}{l}
x \\
y
\end{array}\right)=\left(\begin{array}{c}
c_{m-1} y^{m-1}+c_{m-3} y^{m-3}+\ldots+c_{0} \\
d_{m} y^{m}+d_{m-2} y^{m-2}+\ldots+d_{1} y
\end{array}\right)
$$

on $K\left[x^{1 / t}, x^{-1 / t}, y\right]$ commutes with the $K$-derivation

$$
\alpha\left(\begin{array}{l}
x \\
y
\end{array}\right)=\left(\begin{array}{l}
y \\
h
\end{array}\right)
$$

on $K\left[x^{1 / t}, x^{-1 / t}, y\right]$, then

$$
P_{m}(N)=0 \quad \text { or } \quad N \in\{-1\} \cup\left\{-\frac{k}{k-1} \mid 2 \leqslant k \leqslant \frac{m+1}{2}\right\},
$$

where $N=\operatorname{deg} h$, each $c_{i}, d_{i} \in K\left[x^{1 / t}, x^{-1 / t}\right]$ and $d_{m} \neq 0$.

Proof. Fix $m \geqslant 3$. For $i=0, \ldots, m$, we define $T_{i}(X) \in \mathbb{Z}[X]$ as follows. Let

$$
T_{m}(X)=T_{m-1}(X)=1 .
$$

For $1 \leqslant k \leqslant \frac{m-1}{2}$, let

$$
T_{m-2 k}(X)=X \cdot T_{m-(2 k-1)}(X)-(m-(2 k-2)) \cdot((k-1) \cdot(X+1)+1) \cdot T_{m-(2 k-2)}(X)
$$

and let

$$
T_{m-(2 k+1)}(X)=T_{m-2 k}(X)-(m-(2 k-1)) \cdot k \cdot(X+1) \cdot T_{m-(2 k-1)}(X) .
$$

Let

$$
P_{m}(X)=\left(\frac{m-1}{2} \cdot(X+1)+1\right) \cdot T_{1}(X)-X \cdot T_{0}(X) .
$$

We first prove that

$$
\operatorname{deg} P_{m}(X) \leqslant \frac{m+1}{2} .
$$

We show by induction on $k, 0 \leqslant k \leqslant \frac{m-1}{2}$, that

$$
\operatorname{deg} T_{m-2 k}(X) \leqslant k \text { and } \operatorname{deg} T_{m-(2 k+1)}(X) \leqslant k .
$$

For the base case, $k=0$, we have

$$
\operatorname{deg} T_{m}(X)=\operatorname{deg} T_{m-1}(X)=0 .
$$

For the inductive step, fix $k, 0 \leqslant k<\frac{m-1}{2}$, and assume (33). It follows from (29) and the inductive hypothesis that

$$
\operatorname{deg} T_{m-(2 k+2)}(X) \leqslant k+1,
$$

and it follows from (30) and the inductive hypothesis that

$$
\operatorname{deg} T_{m-(2 k+3)}(X) \leqslant k+1 .
$$

This completes the proof by induction. As a consequence, we have

$$
\operatorname{deg} T_{1}(X) \leqslant \frac{m-1}{2} \quad \text { and } \quad \operatorname{deg} T_{0}(X) \leqslant \frac{m-1}{2} .
$$


Therefore, (32) holds. Next, we show that $P_{m}(X)$ is not the zero polynomial. To this end, we first prove by induction on $k, 0 \leqslant k \leqslant \frac{m-1}{2}$, that

$$
T_{m-2 k}(-1) \neq 0 \quad \text { and } \quad T_{m-(2 k+1)}(-1) \neq 0 .
$$

The base case, $k=0$, is trivial, since $T_{m}(X)=T_{m-1}(X)=1$. For the inductive hypothesis, fix $k$, $0 \leqslant k<\frac{m-1}{2}$, and assume

$$
T_{m-2 k}(-1) \cdot T_{m-(2 k+1)}(-1) \neq 0 .
$$

Equation (30) shows that

$$
T_{m-(2 k+1)}(-1)=T_{m-2 k}(-1) .
$$

Replacing $k$ with $k+1$ in (29) gives us

$$
T_{m-(2 k+2)}(-1)=-1 \cdot T_{m-(2 k+1)}(-1)-(m-2 k) \cdot T_{m-2 k}(-1)=-(m-2 k+1) \cdot T_{m-2 k}(-1) .
$$

Since $k<\frac{m-1}{2}$, it must be that $m-2 k+1 \neq 0$. Now by the inductive hypothesis,

$$
T_{m-(2 k+2)}(-1) \neq 0 \text {. }
$$

Replacing $k$ with $k+1$ in (30) yields

$$
T_{m-(2 k+3)}(-1)=T_{m-(2 k+2)}(-1) \neq 0 .
$$

This completes the proof of (34). By (31), we have

$$
P_{m}(-1)=T_{1}(-1)+T_{0}(-1) .
$$

Replacing $k$ with $\frac{m-1}{2}$ in (30) gives

$$
T_{0}(-1)=T_{1}(-1)
$$

and hence

$$
P_{m}(-1)=2 \cdot T_{1}(-1) \neq 0 .
$$

This completes the proof that $P_{m}(X)$ is not the zero polynomial.

We proceed to show that $P_{m}(X)$ satisfies the remaining property stated in the lemma. Fix $t \in \mathbb{Z}^{\geq 1}$, fix $h \in K\left[x^{1 / t}, x^{-1 / t}\right] \backslash\{0\}$, and define $\alpha$ as in the statement of the lemma. Fix $\beta$ as in the statement of the lemma. Note that $c_{i}$ and $d_{i}$ must satisfy the equations of system $(I I o)_{m}$, with $f$ replaced by $h$. Label these equations as follows:

$$
\begin{array}{ll}
e_{m+1}: & d_{m}^{\prime}=0 \\
e_{m}: & c_{m-1}^{\prime}=d_{m} \\
e_{m-1}: & d_{m-2}^{\prime}+m h d_{m}=h^{\prime} c_{m-1} \\
e_{m-2}: & c_{m-3}^{\prime}+(m-1) h c_{m-1}=d_{m-2} \\
\vdots & \vdots \\
e_{m-(2 k-1)}: & d_{m-2 k}^{\prime}+(m-(2 k-2)) h d_{m-(2 k-2)}=h^{\prime} c_{m-(2 k-1)} \\
e_{m-2 k}: & c_{m-(2 k+1)}^{\prime}+(m-(2 k-1)) h c_{m-(2 k-1)}=d_{m-2 k} \\
e_{m-(2 k+1)}: & d_{m-(2 k+2)}^{\prime}+(m-2 k) h d_{m-2 k}=h^{\prime} c_{m-(2 k+1)} \\
e_{m-(2 k+2)}: & c_{m-(2 k+3)}^{\prime}+(m-(2 k+1)) h c_{m-(2 k+1)}=d_{m-(2 k+2)} \\
\vdots & \vdots \\
e_{0}: & h d_{1}=h^{\prime} c_{0}
\end{array}
$$


Let $N=\operatorname{deg} h$ and let $L=\operatorname{lc}(h)$. Assume that

$$
N \notin\{-1\} \cup\left\{-\frac{k}{k-1} \mid 2 \leqslant k \leqslant \frac{m+1}{2}\right\} .
$$

We first show by induction that for all $k, 0 \leqslant k \leqslant \frac{m-1}{2}$,

$$
\operatorname{deg} d_{m-2 k} \leqslant k(N+1) \quad \text { and } \quad \operatorname{deg} c_{m-(2 k+1)} \leqslant k(N+1)+1 .
$$

We first treat the base case, $k=0$. By equations $e_{m+1}$ and $e_{m}, \operatorname{deg} d_{m} \leqslant 0$ and $\operatorname{deg} c_{m-1} \leqslant 1$.

For the inductive hypothesis, fix $k, 0 \leqslant k<\frac{m-1}{2}$ and assume (35). Consider $e_{m-(2 k+1)}$. By the inductive hypothesis, we have

$$
\operatorname{deg}\left(h d_{m-2 k}\right) \leqslant k(N+1)+N \quad \text { and } \quad \operatorname{deg}\left(h^{\prime} c_{m-(2 k+1)}\right) \leqslant k(N+1)+N .
$$

It follows that

$$
\operatorname{deg} d_{m-(2 k+2)} \leqslant(k+1)(N+1) .
$$

Now consider $e_{m-(2 k+2)}$. By the inductive hypothesis,

$$
\operatorname{deg}\left(h c_{m-(2 k+1)}\right) \leqslant(k+1)(N+1) .
$$

It follows from this and (36) that

$$
\operatorname{deg} c_{m-(2 k+3)} \leqslant(k+1)(N+1)+1 .
$$

This concludes the proof of (35) for all $k, 0 \leqslant k \leqslant \frac{m-1}{2}$.

Define $a_{m}, a_{m-1}, \ldots, a_{0}$ as follows. Let

$$
\begin{gathered}
a_{m-2 k}=\text { the coefficient of } x^{k(N+1)} \text { in } d_{m-2 k}, \\
a_{m-(2 k+1)}=\text { the coefficient of } x^{k(N+1)+1} \text { in } c_{m-(2 k+1)} .
\end{gathered}
$$

Equations $e_{m+1}$ and $e_{m}$ and the requirement that $d_{m} \neq 0$ imply that $a_{m-1}=a_{m}$. Now we prove that, for all $k, 1 \leqslant k \leqslant \frac{m-1}{2}$,

$$
a_{m-(2 k+1)}=\left(a_{m-2 k}-(m-(2 k-1)) \cdot L \cdot a_{m-(2 k-1)}\right) \cdot \frac{1}{k(N+1)+1}
$$

and

$$
a_{m-2 k}=\left(L \cdot N \cdot a_{m-(2 k-1)}-(m-(2 k-2)) \cdot L \cdot a_{m-(2 k-2)}\right) \cdot \frac{1}{k(N+1)} .
$$

Fix $k, 1 \leqslant k \leqslant \frac{m-1}{2}$. By equation $e_{m-(2 k-1)}$, we have

$$
d_{m-2 k}^{\prime}=h^{\prime} c_{m-(2 k-1)}-(m-(2 k-2)) \cdot h \cdot d_{m-(2 k-2)} .
$$

Let us write an equation equating the coefficients of $x^{k(N+1)-1}$ on both sides of (39). First, observe that the coefficient of $x^{k(N+1)-1}$ in $d_{m-2 k}^{\prime}$ is $k(N+1) \cdot a_{m-2 k}$. Next consider $h^{\prime} c_{m-(2 k-1)}$. First consider the case $N \neq 0$. It follows that $\operatorname{deg} h^{\prime}=N-1$. By (35), we have

$$
\operatorname{deg} c_{m-(2 k-1)} \leqslant k(N+1)-N \text {. }
$$

Thus, the coefficient of $x^{k(N+1)-1}$ in $h^{\prime} c_{m-(2 k-1)}$ is $N \cdot L \cdot a_{m-(2 k-1)}$. Now consider the case $N=0$. Either $h^{\prime}=0$, or $h^{\prime} \neq 0$ and $\operatorname{deg} h^{\prime}<N-1$. If $h^{\prime}=0$, then $h^{\prime} c_{m-(2 k-1)}=0$ and the coefficient of $x^{k(N+1)-1}$ in $h^{\prime} c_{m-(2 k-1)}$ is 0 , which is equal to $L \cdot N \cdot a_{m-(2 k-1)}$. If $N=0$ and $h^{\prime} \neq 0$, then, since $\operatorname{deg} h^{\prime}<N-1$ and by (40), the coefficient of $x^{k(N+1)-1}$ in $h^{\prime} c_{m-(2 k-1)}$ is 0 , which is equal to $L \cdot N \cdot a_{m-(2 k-1)}$. Finally, consider $h \cdot d_{m-(2 k-2)}$. Since $\operatorname{deg} h=N$ and, by (35),

$$
\operatorname{deg} d_{m-(2 k-2)} \leqslant k(N+1)-N-1,
$$


we see that the coefficient of $x^{k(N+1)-1}$ in $h \cdot d_{m-(2 k-2)}$ is $L \cdot a_{m-(2 k-2)}$. Since $N \neq-1$, we have $k(N+1) \neq 0$. Thus, equating the coefficients of $x^{k(N+1)-1}$ in (38) yields

$$
a_{m-2 k}=\left(L \cdot N \cdot a_{m-(2 k-1)}-(m-(2 k-2)) \cdot L \cdot a_{m-(2 k-2)}\right) \cdot \frac{1}{k(N+1)} .
$$

By equation $e_{m-2 k}$, we have

$$
c_{m-(2 k+1)}^{\prime}=d_{m-2 k}-(m-(2 k-1)) \cdot h \cdot c_{m-(2 k-1)} .
$$

Let us write an equation equating the coefficients of $x^{k(N+1)}$ on either side of (41). The coefficient of $x^{k(N+1)}$ in $c_{m-(2 k+1)}^{\prime}$ is $(k(N+1)+1) \cdot a_{m-(2 k+1)}$. The coefficient of $x^{k(N+1)}$ in $d_{m-2 k}$ is $a_{m-2 k}$. By (35), we have

$$
\operatorname{deg} c_{m-(2 k-1)} \leqslant k(N+1)-N
$$

and, since $\operatorname{deg} h=N$, the coefficient of $x^{k(N+1)}$ in $h c_{m-(2 k-1)}$ is $L \cdot a_{m-(2 k-1)}$. Since $N \neq-\frac{k+1}{k}$, we have $k(N+1)+1 \neq 0$. Thus, equating the coefficients of $x^{k(N+1)}$ on either side of (41) yields

$$
a_{m-(2 k+1)}=\left(a_{m-2 k}-(m-(2 k-1)) \cdot L \cdot a_{m-(2 k-1)}\right) \cdot \frac{1}{k(N+1)+1} .
$$

This concludes the proof of (37) and (38).

For $i=0, \ldots, m$, define $S_{i} \in \mathbb{Z}$ as follows. Let

$$
S_{m}=S_{m-1}=1 \text {. }
$$

For every $k, 1 \leqslant k \leqslant \frac{m-1}{2}$, let

$$
S_{m-2 k}=k(N+1) \cdot S_{m-(2 k-1)} \quad \text { and } \quad S_{m-(2 k+1)}=(k(N+1)+1) \cdot S_{m-2 k} .
$$

Next, we prove by induction that for all $k, 0 \leqslant k \leqslant \frac{m-1}{2}$, we have

$$
T_{m-2 k}(N)=S_{m-2 k} \cdot \frac{1}{L^{k}} \cdot \frac{1}{a_{m}} \cdot a_{m-2 k} \text { and } T_{m-(2 k+1)}(N)=S_{m-(2 k+1)} \cdot \frac{1}{L^{k}} \cdot \frac{1}{a_{m}} \cdot a_{m-(2 k+1)} .
$$

Recall that by our assumption on the form of $\beta$, we have $a_{m} \neq 0$.

The base case, $k=0$, is proved immediately by noting that $a_{m}=a_{m-1}$ follows from $e_{m+1}$ and $e_{m}$.

For the inductive hypothesis, fix $k, 0 \leqslant k<\frac{m-1}{2}$ and assume (42) holds. We have from (29), (42), and the definition of $S_{i}$ that

$$
\begin{aligned}
T_{m-(2 k+2)}(N) & =N \cdot T_{m-(2 k+1)}(N)-(m-2 k)(k(N+1)+1) \cdot T_{m-2 k}(N) \\
& =N \cdot S_{m-(2 k+1)} \cdot \frac{1}{L^{k}} \cdot \frac{1}{a_{m}} \cdot a_{m-(2 k+1)}-(m-2 k)(k(N+1)+1) \cdot S_{m-2 k} \cdot \frac{1}{L^{k}} \cdot \frac{1}{a_{m}} \cdot a_{m-2 k} \\
& =N \cdot \frac{S_{m-(2 k+2)}}{(k+1)(N+1)} \cdot \frac{1}{L^{k}} \cdot \frac{1}{a_{m}} \cdot a_{m-(2 k+1)}-(m-2 k) \cdot \frac{S_{m-(2 k+2)}}{(k+1)(N+1)} \cdot \frac{1}{L^{k}} \cdot \frac{1}{a_{m}} \cdot a_{m-2 k} \\
& =S_{m-(2 k+2)} \cdot \frac{1}{L^{k}} \cdot \frac{1}{a_{m}} \cdot\left(N a_{m-(2 k+1)}-(m-2 k) a_{m-2 k}\right) \cdot \frac{1}{(k+1)(N+1)} .
\end{aligned}
$$

From (38) with $k$ replaced by $k+1$, we see that

$$
T_{m-(2 k+2)}(N)=S_{m-(2 k+2)} \cdot \frac{1}{L^{k+1}} \cdot \frac{1}{a_{m}} \cdot a_{m-(2 k+2)} \cdot
$$

We have from (30), (43), (42), and the definition of $S_{i}$ that

$$
\begin{aligned}
& T_{m-(2 k+3)}(N)=T_{m-(2 k+2)}(N)-(m-(2 k+1)) \cdot(k+1)(N+1) \cdot T_{m-(2 k+1)}(N) \\
& =S_{m-(2 k+2)} \cdot \frac{1}{L^{k+1}} \cdot \frac{1}{a_{m}} \cdot a_{m-(2 k+2)}-(m-(2 k+1)) \cdot(k+1)(N+1) \cdot S_{m-(2 k+1)} \cdot \frac{1}{L^{k}} \cdot \frac{1}{a_{m}} \cdot a_{m-(2 k+1)} \\
& =S_{m-(2 k+3)} \cdot \frac{1}{L^{k+1}} \cdot \frac{1}{a_{m}} \cdot\left(a_{m-(2 k+2)}-L(m-(2 k+1)) a_{m-(2 k+1)}\right) \cdot \frac{1}{(k+1)(N+1)+1} .
\end{aligned}
$$


From (37) with $k$ replaced by $k+1$, we see that

$$
T_{m-(2 k+3)}(N)=S_{m-(2 k+3)} \cdot \frac{1}{L^{k+1}} \cdot \frac{1}{a_{m}} \cdot a_{m-(2 k+3)} \cdot
$$

This completes the proof of (42).

Now we show that $P_{m}(N)=0$. Using $k=\frac{m-1}{2}$ in (42) and $S_{0}=\left(\frac{m-1}{2}(N+1)+1\right) S_{1}$, we have

$$
\begin{aligned}
P_{m}(N) & =\left(\frac{m-1}{2}(N+1)+1\right) \cdot T_{1}(N)-N \cdot T_{0}(N) \\
& =\left(\frac{m-1}{2}(N+1)+1\right) S_{1} \cdot \frac{1}{L^{(m-1) / 2}} \cdot \frac{1}{a_{m}} \cdot a_{1}-N \cdot S_{0} \cdot \frac{1}{L^{(m-1) / 2}} \cdot \frac{1}{a_{m}} \cdot a_{0}=\frac{S_{0}}{L^{(m-1) / 2}} \cdot \frac{1}{a_{m}} \cdot\left(a_{1}-N a_{0}\right) .
\end{aligned}
$$

Consider equation $e_{0}$ :

$$
h d_{1}=h^{\prime} c_{0} .
$$

Equating the coefficients of $x^{(N+1)((m-1) / 2)+N}$ in $e_{0}$, recalling (35), yields

$$
a_{1}=N a_{0} .
$$

We conclude that $P_{m}(N)=0$.

Lemma 3.7. For every positive integer $k$, the $K$-derivation

$$
\alpha\left(\begin{array}{l}
x \\
y
\end{array}\right)=\left(\begin{array}{c}
y \\
x^{-\frac{2 k+1}{2 k-1}}
\end{array}\right)
$$

of the ring $K\left[x^{-\frac{1}{2 k-1}}, x^{\frac{1}{2 k-1}}, y\right]$ commutes with the $K$-derivation

$$
\beta\left(\begin{array}{l}
x \\
y
\end{array}\right)=\left(\begin{array}{c}
\sum_{l=0}^{k} a_{2(k-l)} x^{1+\left(1-\frac{2 k+1}{2 k-1}\right) l} y^{2(k-l)} \\
\sum_{l=0}^{k} a_{2(k-l)+1} x^{\left(1-\frac{2 k+1}{2 k-1}\right) l} y^{2(k-l)+1}
\end{array}\right),
$$

where the $a_{i} \in K$ are defined recursively as follows: $a_{2 k+1} \in K \backslash\{0\}$ is arbitrary, $a_{2 k}=a_{2 k+1}$, and for $0<l \leqslant k$,

$$
\begin{aligned}
a_{2(k-l)+1} & =\left(-\frac{2 k+1}{2 k-1} a_{2(k-l)+2}-(2(k-l)+3) a_{2(k-l)+3}\right)\left(\left(1-\frac{2 k+1}{2 k-1}\right) l\right)^{-1} \\
a_{2(k-l)} & =\left(a_{2(k-l)+1}-(2(k-l)+2) a_{2(k-l)+2}\right)\left(\left(1-\frac{2 k+1}{2 k-1}\right) l+1\right)^{-1} .
\end{aligned}
$$

Proof. We first show that

$$
\beta(\alpha(x))=\alpha(\beta(x)) \text {. }
$$

We have $\beta(\alpha(x))=\beta(y)$. Note that, in $\beta(y)$, only odd powers of $y$ with exponents less than or equal to $2 k+1$ appear, and for all $l, 0 \leqslant l \leqslant k$, the coefficient of $y^{2(k-l)+1}$ is

$$
a_{2(k-l)+1} x^{\left(1-\frac{2 k+1}{2 k-1}\right) l} \text {. }
$$

In $\alpha(\beta(x))$, only odd powers of $y$ with exponents less than or equal to $2 k+1$ appear. The coefficient of $y^{2 k+1}$ is $a_{2 k}$, which equals $a_{2 k+1}$, which is the coefficient of $y^{2 k+1}$ in $\beta(\alpha(x))$. For all $l, 1 \leqslant l \leqslant k$, the coefficient of $y^{2(k-l)+1}$ in $\alpha(\beta(x))$ is

$$
a_{2(k-l)} x^{\left(1-\frac{2 k+1}{2 k-1}\right) l}\left(1+\left(1-\frac{2 k+1}{2 k-1}\right) l\right)+a_{2(k-l)+2} x^{\left(1-\frac{2 k+1}{2 k-1}\right) l}(2(k-l)+2) .
$$

By the definition of $a_{2(k-l)}$, this equals (44). Now we show that

$$
\beta(\alpha(y))=\alpha(\beta(y)) .
$$

We have

$$
\beta(\alpha(y))=\beta\left(x^{-\frac{2 k+1}{2 k-1}}\right)=-\frac{2 k+1}{2 k-1} x^{-\frac{2 k+1}{2 k-1}-1} \beta(x) .
$$


This expression contains only even powers of $y$ from $y^{0}$ to $y^{2 k}$. For all $l, 0 \leqslant l \leqslant k$, the coefficient of $y^{2(k-l)}$ in $\beta(\alpha(y))$ is

$$
-\frac{2 k+1}{2 k-1} a_{2(k-l)} x^{\left(1-\frac{2 k+1}{2 k-1}\right) l-\frac{2 k+1}{2 k-1}}
$$

We see that $\alpha(\beta(y))$ contains only even powers of $y$ from $y^{0}$ to $y^{2 k}$. For $l<k$, the coefficient of $y^{2(k-l)}$ in $\alpha(\beta(y))$ is

$$
a_{2(k-l)+1} x^{\left(1-\frac{2 k+1}{2 k-1}\right) l-\frac{2 k+1}{2 k-1}}(2(k-l)+1)+a_{2(k-l)-1} x^{\left(1-\frac{2 k+1}{2 k-1}\right) l-\frac{2 k+1}{2 k-1}}\left(1-\frac{2 k+1}{2 k-1}\right)(l+1) .
$$

By definition,

$$
a_{2(k-l)-1}=\left(-\frac{2 k+1}{2 k-1} a_{2(k-l)}-(2(k-l)+1) a_{2(k-l)+1}\right)\left(\left(1-\frac{2 k+1}{2 k-1}\right)(l+1)\right)^{-1} .
$$

Hence, the coefficient of $y^{2(k-l)}$ in $\alpha(\beta(y))$ is (45). The coefficient of $y^{0}$ in $\alpha(\beta(y))$ is

$$
a_{1} x^{\left(1-\frac{2 k+1}{2 k-1}\right) k-\frac{2 k+1}{2 k-1}} \text {. }
$$

It remains to show that

$$
a_{1}=-\frac{2 k+1}{2 k-1} a_{0}
$$

This is an immediate consequence of the following lemma.

Lemma 3.8. In the notation of Lemma 3.7, for all $l, 0 \leqslant l \leqslant k$,

$$
\frac{2 k+1}{2 k-1} a_{2(k-l)}=\frac{2(k-l)+1}{2(k-l)-1} a_{2(k-l)+1} .
$$

Proof. We proceed by induction on $l$. The base case $l=0$ is immediate, since by definition $a_{2 k}=$ $a_{2 k+1}$. For the inductive hypothesis, fix $l<k$ and assume

$$
\frac{2 k+1}{2 k-1} a_{2(k-l)}=\frac{2(k-l)+1}{2(k-l)-1} a_{2(k-l)+1} \text {. }
$$

We want to show that

$$
\frac{2 k+1}{2 k-1} a_{2(k-l)-2}=\frac{2(k-l)-1}{2(k-l)-3} a_{2(k-l)-1} .
$$

The left-hand side of (47) is, by the definition of $a_{2(k-l)-2}$,

$$
\frac{2 k+1}{2 k-1} \cdot \frac{\left(a_{2(k-l)-1}-2(k-l) a_{2(k-l)}\right)}{\left(1-\frac{2 k+1}{2 k-1}\right)(l+1)+1} .
$$

By the definition of $a_{2(k-l)-1}$, this equals

$$
\frac{2 k+1}{2 k-1} \cdot \frac{\left(\frac{-\frac{2 k+1}{2 k-1} a_{2(k-l)}-(2(k-l)+1) a_{2(k-l)+1}}{\left(1-\frac{2 k+1}{2 k-1}\right)(l+1)}-2(k-l) a_{2(k-l)}\right)}{\left(1-\frac{2 k+1}{2 k-1}\right)(l+1)+1} .
$$

By the inductive hypothesis, this is equal to

$$
\frac{\left(\frac{-\frac{2 k+1}{2 k-1} \cdot \frac{2(k-l)+1}{2(k-l)-1}-\frac{2 k+1}{2 k-1}(2(k-l)+1)}{\left(1-\frac{2 k+1}{2 k-1}\right)(l+1)}-2(k-l) \frac{2(k-l)+1}{2(k-l)-1}\right)}{\left(1-\frac{2 k+1}{2 k-1}\right)(l+1)+1} \cdot a_{2(k-l)+1} .
$$


The right-hand side of (47) is, using the definition of $a_{2(k-l)-1}$,

$$
\frac{2(k-l)-1}{2(k-l)-3} \cdot \frac{-\frac{2 k+1}{2 k-1} a_{2(k-l)}-(2(k-l)+1) a_{2(k-l)+1}}{\left(1-\frac{2 k+1}{2 k-1}\right)(l+1)} .
$$

By the inductive hypothesis, this equals

$$
\frac{2(k-l)-1}{2(k-l)-3} \frac{-\frac{2(k-l)+1}{2(k-l)-1}-(2(k-l)+1)}{\left(1-\frac{2 k+1}{2 k-1}\right)(l+1)} \cdot a_{2(k-l)+1},
$$

which is equal to (48), as a computation shows.

By letting $l=k$ in Lemma 3.8, we see that (46) holds.

Lemma 3.9. For every

- positive integer $t$,

- $h \in K\left[x^{1 / t}, x^{-1 / t}\right] \backslash\{0\}$,

if there exists a $K$-derivation $\beta$ on $K\left[x^{1 / t}, x^{-1 / t}, y\right]$ such that

- $\beta$ commutes with the $K$-derivation

$$
\alpha\left(\begin{array}{l}
x \\
y
\end{array}\right)=\left(\begin{array}{l}
y \\
h
\end{array}\right)
$$

on $K\left[x^{-1 / t}, x^{1 / t}, y\right]$ and

- $\beta$ is of the form

$$
\beta\left(\begin{array}{l}
x \\
y
\end{array}\right)=\left(\begin{array}{c}
c_{m-1} y^{m-1}+c_{m-3} y^{m-3}+\ldots+c_{0} \\
d_{m} y^{m}+d_{m-2} y^{m-2}+\ldots+d_{1} y
\end{array}\right)
$$

where $m \geqslant 3$ is odd, $c_{i}, d_{i} \in K\left[x^{-1 / t}, x^{1 / t}\right]$, and $d_{m} \neq 0$,

then

$$
N:=\operatorname{deg} h \in S \cup T
$$

where

$$
\begin{gathered}
S=\{1\} \cup\left\{-\frac{2 k+1}{2 k-1} \mid k \in \mathbb{Z}, 1 \leqslant k \leqslant \frac{m-1}{2}\right\}, \\
T=\{-1\} \cup\left\{-\frac{k}{k-1} \mid k \in \mathbb{Z}, k \geqslant 2\right\} .
\end{gathered}
$$

Proof. Fix $t \in \mathbb{Z}^{\geq 1}$. Fix $h \in K\left[x^{-1 / t}, x^{1 / t}\right] \backslash\{0\}$ and hence $\alpha$ of the form stated in the lemma. Let $N=\operatorname{deg} h$ and assume $N \notin T$. Suppose a $K$-derivation $\beta$ satisfying the properties stated in the lemma exists and let $m$ be the least odd integer greater than or equal to 3 such that there exists such a $\beta$. By Lemma 3.6, $P_{m}(N)=0$, and $P_{m}$ has at most $\frac{m+1}{2}$ zeros. We show that these zeros are exactly the elements of $S$.

We show that $P_{m}(1)=0$. The $K$-derivations

$$
\partial_{1}\left(\begin{array}{l}
x \\
y
\end{array}\right)=\left(\begin{array}{l}
y \\
x
\end{array}\right) \quad \text { and } \quad \partial_{2}\left(\begin{array}{l}
x \\
y
\end{array}\right)=\left(\begin{array}{l}
x \\
y
\end{array}\right)
$$

on $K\left[x, x^{-1}, y\right]$ commute and $\partial_{1}$ has the form of $\alpha$ in the statement of Lemma 3.6. The polynomial $r:=y^{2}-x^{2}$ is a first integral of $\partial_{1}$, and so $r^{(m-1) / 2} \partial_{2}$ is a $K$-derivation commuting with $\partial_{1}$ of the form of $\beta$ in the statement of Lemma 3.6. Therefore, by Lemma 3.6, $P_{m}(1)=0$. 
We show that, for all $k, 1 \leqslant k \leqslant \frac{m-1}{2}$,

$$
P_{m}\left(-\frac{2 k+1}{2 k-1}\right)=0
$$

Fix $k$. Let $K$-derivations $\partial_{1}$ and $\partial_{2}$ on $K\left[x^{\frac{1}{2 k-1}}, x^{-\frac{1}{2 k-1}}, y\right]$ be defined as $\alpha$ and $\beta$ are in Lemma 3.7. Now

$$
r=y^{2}+2\left(\frac{2 k-1}{2}\right) x^{-2 /(2 k-1)}
$$

is a first integral of $\partial_{1}$. Note that $\operatorname{deg}_{y} \partial_{2}(y)=2 k+1$. Now $r^{(m-(2 k+1)) / 2} \partial_{2}$ is a derivation commuting with $\partial_{1}$ of the form of $\beta$ of Lemma 3.6. Hence, we have (49).

The set $S$ consists of $\frac{m+1}{2}$ elements, and we have shown that each is a zero of $P_{m}$, which is nonzero of degree at most $\frac{m+1}{2}$. It follows that $S$ is exactly the zero set of $P_{m}$.

Corollary 3.4. It is impossible that $p$ is an odd integer greater than or equal to 3.

Proof. Suppose $p \geqslant 3$ and $p$ is odd. Let $N=\operatorname{deg} f$. Recall that $p=\operatorname{deg}_{y} \gamma_{2}$. Consider Lemma 3.9. Since the extensions of $\delta$ and $\gamma_{2}$ to $K$-derivations on $K\left[x, x^{-1}, y\right]$ are of the forms of $\alpha$ and $\beta$, it follows that $N \in S \cup T$. Since $N$ is assumed to be an integer greater than or equal to 2 , this is a contradiction.

This finishes the proof of Theorem 3.1.

\section{ACKNOWLEDGEMENTS}

This work was partially supported by the NSF grants CCF-0952591, DMS-1700336, DMS1606334, by the NSA grant \#H98230-15-1-0245, by CUNY CIRG \#2248, and by PSC-CUNY grants \#69827-00 47 and \#60456-00 48. The authors are grateful to the CCiS at CUNY Queens College for the computational resources.

\section{REFERENCES}

[1] V. Amel'kin. Isochronism of a center for two-dimensional analytic differential systems. Differential Equations, 13(6):667-674, 1977.

[2] C. Chicone and M. Jacobs. Bifurcation of critical periods for plane vector fields. Transactions of the American Mathematics Society, 312(2):433-486, 1989. URL http://dx.doi.org/10.1090/s0002-9947-1989-0930075-2.

[3] A. G. Choudhury and P. Guha. On commuting vector fields and Darboux functions for planar differential equations. Lobachevskii Journal of Mathematics, 34(3):212-226, 2013. URL http://dx.doi.org/10.1134/S1995080213030049.

[4] A. Cima, F. Mañosas, and J. Villadelprat. Isochronicity for several classes of Hamiltonian systems. Journal of Differential Equations, 157(2):373-413, 1999. URL http://dx.doi.org/10.1006/jdeq.1999.3635.

[5] J. Giné and M. Grau. Linearizability and integrability of vector fields via commutation. Journal of Mathematical Analysis and Applications, 319(1):326-332, 2006. URL http://dx.doi.org/10.1016/j.jmaa.2005.10.017.

[6] I. Kaplansky. An Introduction to Differential Algebra. Hermann, Paris, 1957.

[7] E. Kolchin. Differential Algebra and Algebraic Groups. Academic Press, New York, 1973.

[8] J. Li and $\mathrm{X}$. Du. Pairwise commuting derivations of polynomial rings. Linear Algebra and its Applications, 436(7):2375-2379, $2012 . \quad$ URL http://dx.doi.org/10.1016/j.laa.2011.10.007. 
[9] A. Nowicki. Polynomial derivations and their rings of constants. Uniwersytet Mikołaja Kopernika, Toruń, 1994.

[10] A. Petravchuk. On pairs of commuting derivations of the polynomial ring in two variables. Linear Algebra and its Applications, 433(3):574-579, 2010. URL http://dx.doi.org/10.1016/j.laa.2010.03.030.

[11] M. Sabatini. Characterizing isochronous centres by Lie brackets. Differential Equations and Dynamical Systems, 5(1):91-99, 1997.

[12] M. Villarini. Regularity properties of the period function near a center of a planar vector field. Nonlinear Analysis: Theory, Methods \& Applications, 19(8):787-803, 1992. URL http://dx.doi.org/10.1016/0362-546X (92) 90222-z.

[13] E. Volokitin and V. Ivanov. Isochronicity and commutation of polynomial vector fields. Siberian Mathematical Journal, 40(1):23-38, 1999. URL http://dx.doi.org/10.1007/BF02674287.

CUny Bronx Community College, Department of Mathematics and Computer Science, 2155 UNIVERSITY AVENUE BRONX, NY 10453

E-mail address: joel.nagloo@bcc. cuny. edu

URL: http: //fsw01.bcc.cuny.edu/joel.nagloo/

Cuny Queens College, Department of Mathematics, 65-30 Kissena Blvd, Queens, Ny 11367 and CUNy Graduate Center, Ph.D. Programs in Mathematics and Computer Science, 365 Fifth AVEnUE, NEW YORK, NY 10016

E-mail address: aovchinnikov@qc . cuny . edu

URL: http://qc.edu/ aovchinnikov/

Cuny Graduate Center, Ph.D. Program in Mathematics, 365 Fifth Avenue, New York, Ny 10016

E-mail address: pthompson@gradcenter. cuny .edu

URL: http: / / peterthompsonmath.wordpress.com 